\title{
Congenital arch malformation: a review of morphology and surgical management of circumflex aortic arch
}

\author{
Vivian Bader ${ }^{1}$, W. Brodie Knight ${ }^{2}$, Mark H. Danton ${ }^{1,3}$, Ed Peng ${ }^{1,3}$ \\ 1Department of Paediatric Cardiac Surgery, Royal Hospital for Children, Glasgow, Scotland G51 4TF, United Kingdom. \\ 2Paediatric Cardiology department, Scottish Paediatric Cardiac Services, Royal Hospital for Children, Glasgow, Scotland G51 \\ 4TF, United Kingdom. \\ ${ }^{3}$ College of Medical, Veterinary and Life Sciences, School of Medicine, Dentistry \& Nursing, University of Glasgow, Glasgow G12 \\ $8 \mathrm{QQ}$, United Kingdom.
}

Correspondence to: Mr. Ed Peng, Department of Paediatric Cardiac Surgery, Royal Hospital for Children, Queen Elizabeth University Hospital, New Office Block, 1345 Govan Rd, Glasgow, Scotland G51 4TF, United Kingdom.

E-mail: edwkpeng@doctors.org.uk

How to cite this article: Bader V, Knight WB, Danton MH, Peng E. Congenital arch malformation: a review of morphology and surgical management of circumflex aortic arch. Vesse/ P/us 2020;4:34. http://dx.doi.org/10.20517/2574-1209.2020.36

Received: 3 Aug 2020 First Decision: 21 Sep 2020 Revised: 28 Sep 2020 Accepted: 12 Oct 2020 Published: 13 Nov 2020

Academic Editor: Lizhong Sun, Junmin Zhu, Haiou Hu Copy Editor: Cai-Hong Wang Production Editor: Jing Yu

\begin{abstract}
Circumflex aorta is an unusual form of congenital arch malformation with a retroesophageal arch segment. Circumflex aorta crosses the midline behind the oesophagus and above the level of carina to become continuous with the proximal descending aorta on the opposite side to form a true vascular ring with the arterial ligament. The term is often used to refer to the more common variant of circumflex aorta with a retroesophageal right-sided aortic arch with a left-sided descending aorta and left-sided ligamentum. Its mirror-form with a retroesophageal left-sided aortic arch is much rarer. Although originally described without obstruction, it may occur in association with aortic arch hypoplasia, adding to its clinical burden. This article describes the morphology of circumflex aorta and its clinical presentation, and provides review of surgical management of circumflex aortic arch.
\end{abstract}

Keywords: Congenital, arch malformation, morphology, circumflex aorta, surgery

\section{INTRODUCTION}

Circumflex aorta is an unusual form of congenital aortic arch anomaly with a retroesophageal arch segment. The term is most commonly used to refer to an aorta that passes to the right of the trachea (i.e., 
a right aortic arch), which crosses the midline behind the oesophagus above the airway carina, to become continuous with the proximal descending thoracic aorta on the opposite side ${ }^{[1,2]}$. In the majority of cases an associated Kommerell's diverticulum with an aberrant left subclavian artery also gives rise to a leftsided arterial duct, which forms a complete vascular ring. Simple vascular ring division may not fully address all the deleterious effects of circumflex aorta, and a major aortic arch uncrossing procedure may be required ${ }^{[1-4]}$. The aortic arch is often unobstructed or presents with variable degrees of obstruction from hypoplasia to near-interruption requiring major arch repair ${ }^{[5-7]}$. When there is also obstruction of the proximal aberrant subclavian, it can be confused clinically with aortic dissection in adult patients ${ }^{[8]}$. The mirror image of the usual form of circumflex aorta, with left aortic arch and right descending aorta, is very rare, and may require a different surgical approach ${ }^{[7,9]}$.

\section{MORPHOLOGY AND EMBRYOGENESIS}

Although the term circumflex aorta was used in the literature as early as 1960, morphological arch descriptions similar to circumflex aorta can be traced back to late 19th century ${ }^{[10-14]}$. Circumflex aorta with a right aortic arch is much more common than with a left aortic $\operatorname{arch}^{[13,15]}$.

\section{Right aortic arch}

A circumflex aorta with a right aortic arch refers to a retroesophageal right aortic arch, a left-sided descending thoracic aorta, and a left-sided ligamentum arteriosum. This aortic arch passes over the right main bronchus to the right of trachea and oesophagus. It then crosses the midline behind the oesophagus, and anterior to the spine in the upper mediastinum or above the level of the carina, to join the proximal end of left sided descending aorta. Usually the distal portion of the embryological left dorsal aortic arch forms a Kommerell's diverticulum which gives rise to an aberrant left subclavian artery. A left-sided arterial duct or ligament connects the base of the aberrant subclavian artery and the proximal left pulmonary artery, thus forming a complete vascular ring ${ }^{[10,12]}$. In usual circumflex aortic arch, the order of origin of the arch vessels is: (1) left common carotid artery; (2) right common carotid; (3) right subclavian; (4) from the proximal descending aorta, an aberrant left subclavian artery [Figure 1].

\section{Left aortic arch}

A circumflex aorta with a left aortic arch is a rarer variant of this anomaly and was first reported by Paul (1948). It mirrors the circumflex aorta with right aortic arch: left-sided aortic arch, right-sided descending aorta and right-sided arterial duct or ligament ${ }^{[7,11,15-22]}$. The retroesophageal transverse arch will cross the midline from left to right.

The head and neck arteries arise sequentially as follows: (1) right common carotid; (2) left common carotid; (3) left subclavian, and from proximal descending aorta; (4) an aberrant right subclavian artery [Figure 2].

\section{Retroesophageal transverse arch}

A retroesophageal transverse arch, which crosses the spine at T2-T3 level above the tracheal carina is the hallmark of circumflex aorta. The proximal descending aorta is located contralateral to the aortic arch, giving rise to the characteristic course of a circumflex arch. The thoracic descending aorta can course back to the other side of the spine before it descends via the aortic hiatus at the diaphragm to form the abdominal aorta ${ }^{[7,10,12]}$.

\section{Aberrant subclavian artery and Kommerell diverticulum}

A circumflex aortic arch is associated with an aberrant subclavian artery where a bulbous dilatation can often be found at its origin. This subclavian artery arises aberrantly from the proximal descending aorta, rather than the aortic $\operatorname{arch}^{[23]}$. It is at this base of aberrant subclavian where the arterial duct is connected to the pulmonary artery. After involution of ductus arteriosus, the arterial ligament remains connected 


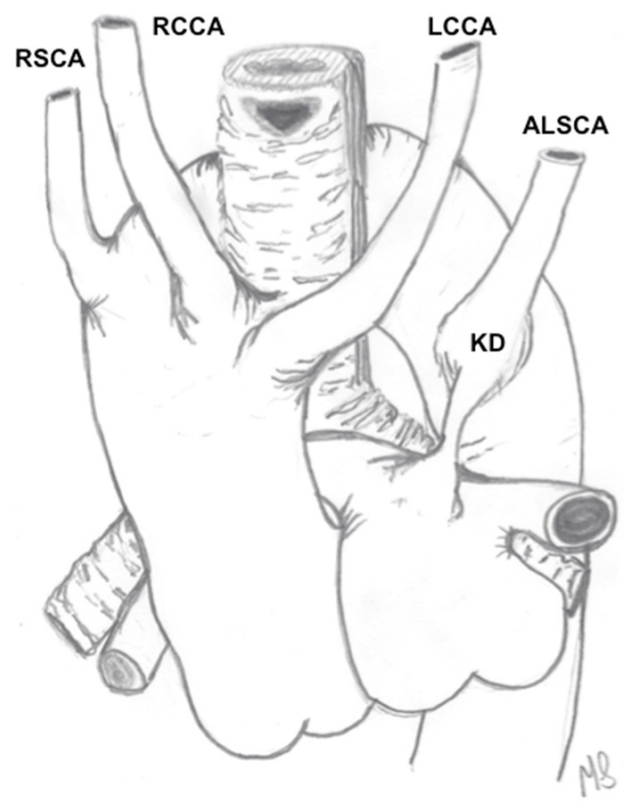

Figure 1. Circumflex aorta with retroesophageal right aortic arch, left sided descending aorta and left ductal ligament with the following branches: left common carotid artery (LCCA), right common carotid artery (RCCA), right subclavian artery (RSCA), and aberrant left subclavian artery (ALSCA) arising from Kommerell's diverticulum (KD)

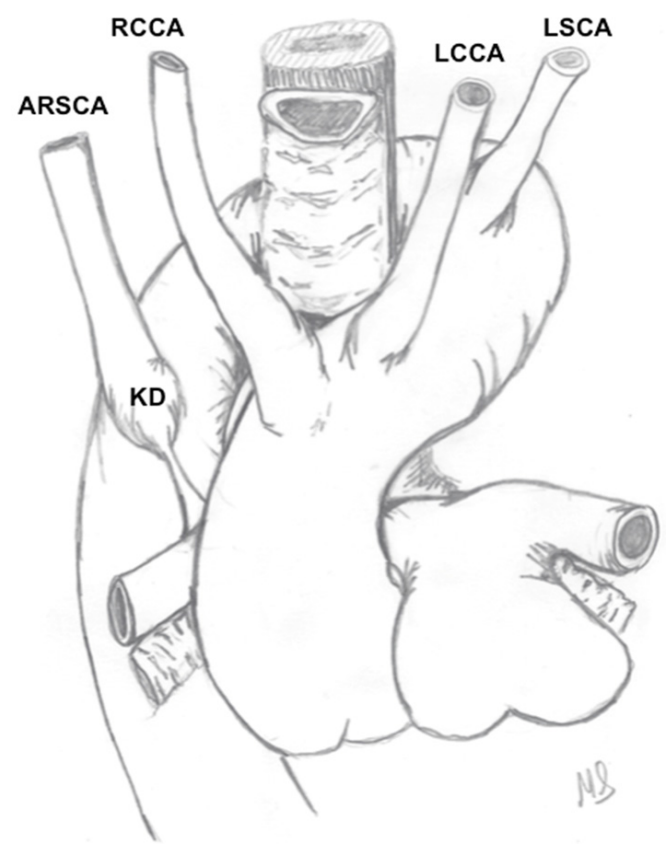

Figure 2. Circumflex aorta with retroesophageal left-sided aortic arch, right sided descending aorta and left-sided ductal ligament with the following branches: right common carotid artery (RCCA), left common carotid artery (LCCA), left subclavian artery (LSCA) and aberrant right subclavian artery (ARSCA) arising from Kommerell's diverticulum (KD)

to the base of this subclavian artery, forming a vascular ring. Named after Kommerell who described the anomaly as an incidental radiographic finding in 1936, this bulbous dilatation is a remnant of fourth dorsal aortic $\operatorname{arch}^{[24-26]}$. A significant Kommerell's diverticulum is one which is at least 1.5 times the size of distal subclavian artery ${ }^{[4]}$. Abnormal histology with cystic medal necrosis in the aortic diverticulum has been described in young children, which explains predilection to form an aneurysm with risk of rupture and dissection later in life ${ }^{[24,27]}$. 
A Kommerell's diverticulum or aberrant subclavian artery is not a constant feature in all reported circumflex aortic arch. Instead of bulbous dilatation of the base of aberrant subclavian, stenosis and obstruction at its origin has also been described in circumflex aortic $\operatorname{arch}^{[8]}$. However, this may represent a ductal tissue constriction that results in "coarctation" of the junction between the Kommerell's diverticulum and the origin of the embryological left subclavian artery. A subclavian with usual origin from the innominate trunk has also been described in circumflex aortic $\operatorname{arch}^{[12,15]}$.

\section{Concomitant lesions}

Aortic arch obstruction with a hypoplastic retroesophageal segment is the most important associated lesion. The retroesophageal arch segment is usually of adequate caliber, but significant hypoplasia can occur particularly with concomitant ventricular septal defect, which can mimic interruption ${ }^{[6,28,29]}$. When arch hypoplasia is present, it can be tubular with a long segment, or tortuous, extending from the proximal to distal transverse arch as it courses around the side of the trachea and back of the oesophagus ${ }^{[5-7,28]}$. A long hypoplastic retroesophageal segment can be mistaken as an Abbott artery which is an anomalous artery arising from the posterior wall of the aortic arch or others in coarctation of the aorta ${ }^{[30-32]}$.

Concomitant intra-cardiac lesions, commonly VSD, can also be present with circumflex $\operatorname{arch}^{[6,14,33]}$. Other reported cardiac lesions include atrial septal defect and bicuspid aortic valve $e^{[14,33]}$. Less commonly, Tetralogy of Fallot or double outlet RV, a lesion with a higher frequency of right aortic arch than in the normal population, had also been reported in circumflex aorta ${ }^{[15]}$; as well as left heart hypoplasia ${ }^{[5,34]}$.

\section{Vascular ring variants resembling circumflex aorta}

Following repair of double aortic arch where the smaller left arch is divided [Figure 3A-C], the preserved retroesophageal arch segment will resemble a circumflex $\operatorname{arch}^{[35]}$. A completely atretic segment [Figure 3D] can be present between the left common carotid and subclavian arteries in the setting of a double aortic arch with a dominant right ${ }^{[36-38]}$. They are strictly speaking, by morphology criteria, a double aortic arch, but functionally resemble a circumflex arch with a high retroesophageal segment above the tracheal carina ${ }^{[36]}$. In true circumflex arch, there is no connecting segment between these two neck vessels [Figure 3A]. Crosssectional imaging does not differentiate between them.

A common variant of vascular ring is a right aortic arch with aberrant subclavian artery and Kommerell diverticulum and left sided arterial ligament (Neuhauser anomaly) [Figure 4]. It shares the same vascular arrangement, in terms of neck vessel origins, with a circumflex aorta, and the latter may be considered as an extreme form of this common variant of right aortic arch. However, there are some important differences as follows: (1) the retroesophageal component is the Kommerell's diverticulum; whereas in circumflex aorta, this aortic diverticulum is to the left of the oesophagus; and (2) the proximal descending aorta is on the right of the spine, i.e., the same side as the arch. Therefore, its transverse arch does not cross behind oesophagus. Both are closely related, but this common variant of right aortic arch is not functionally a circumflex aorta and is likely to have different embryogenesis.

\section{Embryology}

The developmental aetiology of the circumflex arch is unclear. Genetic associations with right arch include a higher prevalence of chromosome 22q11 microdeletions 3,8 and the syndromes that are now known to be associated with this genetic defect, such as DiGeorge, velo-cardio-facial syndrome and conotruncal anomaly face syndrome. The Rathke diagram [Figure 5], based on work of the renowned embryologist, Martin Heinrich Rathke (1793-1860) is classically used to understand the normal development of aortic arch and its branches ${ }^{[39]}$. During the development of the normal aortic arch, there is involution of the right fourth and sixth aortic arches and of the right dorsal aorta (dorsal aortic root segment 8) distal to the right subclavian artery. In normal development, the dorsal aorta that persists is the one on the same side of the 

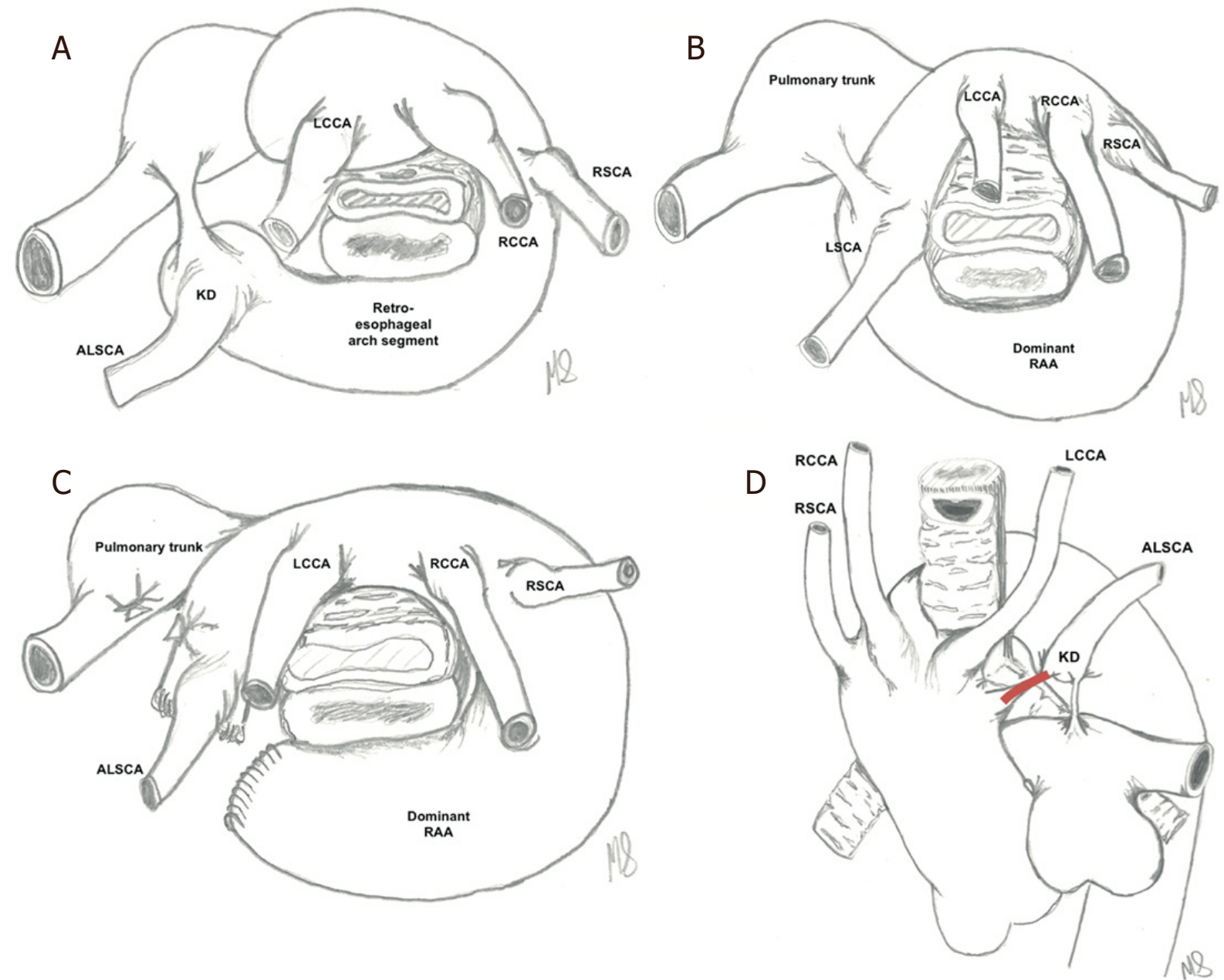

Figure 3. Comparison between $A$ : circumflex aorta with right aortic arch; $B$ : double aortic arch with dominant right aortic arch (dominant RAA) with right common carotid (RCCA) right subclavian artery (RSCA), left common carotid artery (LCCA) and the left subclavian artery (LSCA); C: post-surgical division of the non-dominant distal left aortic arch in a patient with double aortic arch; D: double aortic arch with atretic left segment marked in red with the aberrant left subclavian (ALSCA) arising from Kommerell's diverticulum (KD)
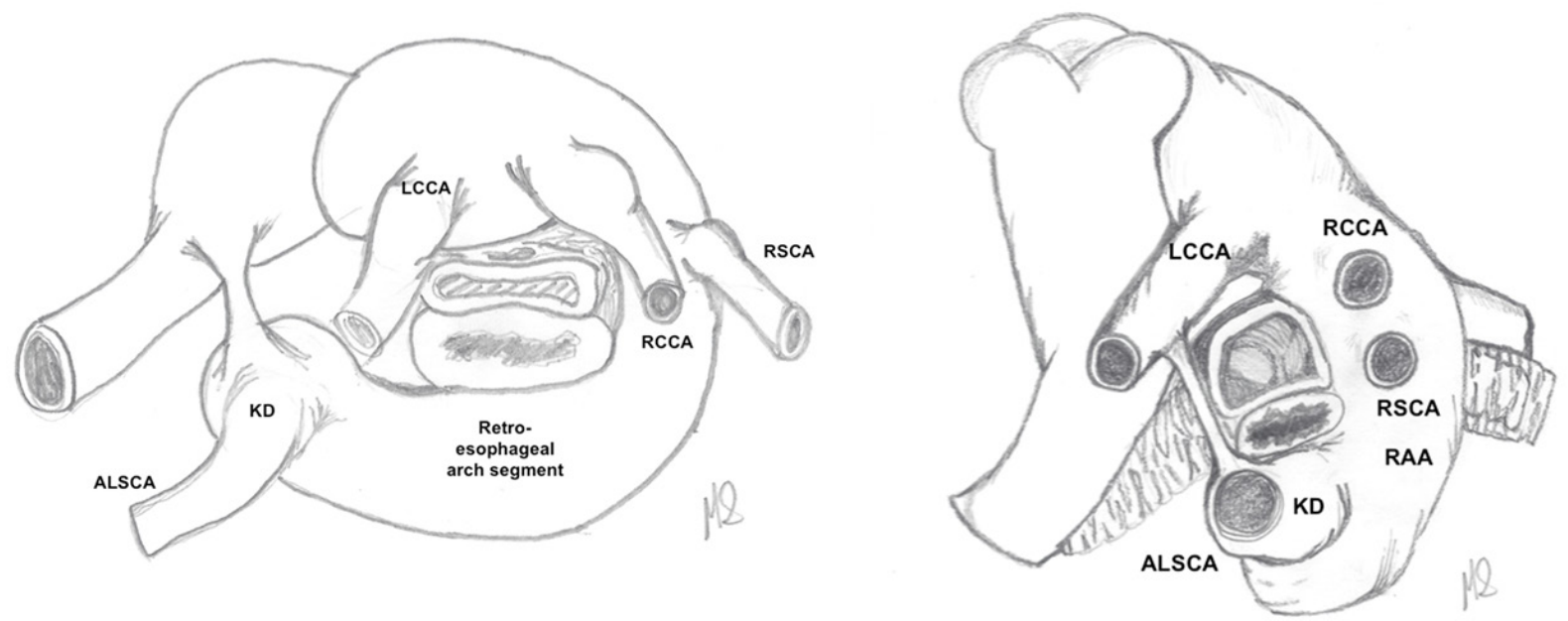

Figure 4. Comparison between circumflex right aortic arch and common variant of right aortic arch with retroesophageal aberrant left subclavian artery (ALSCA) arising from Kommerell diverticulum (KD). Head and neck vessels are left common carotid (LCCA), right common carotid artery (RCCA), right subclavian artery (RSCA), and the ALSCA 


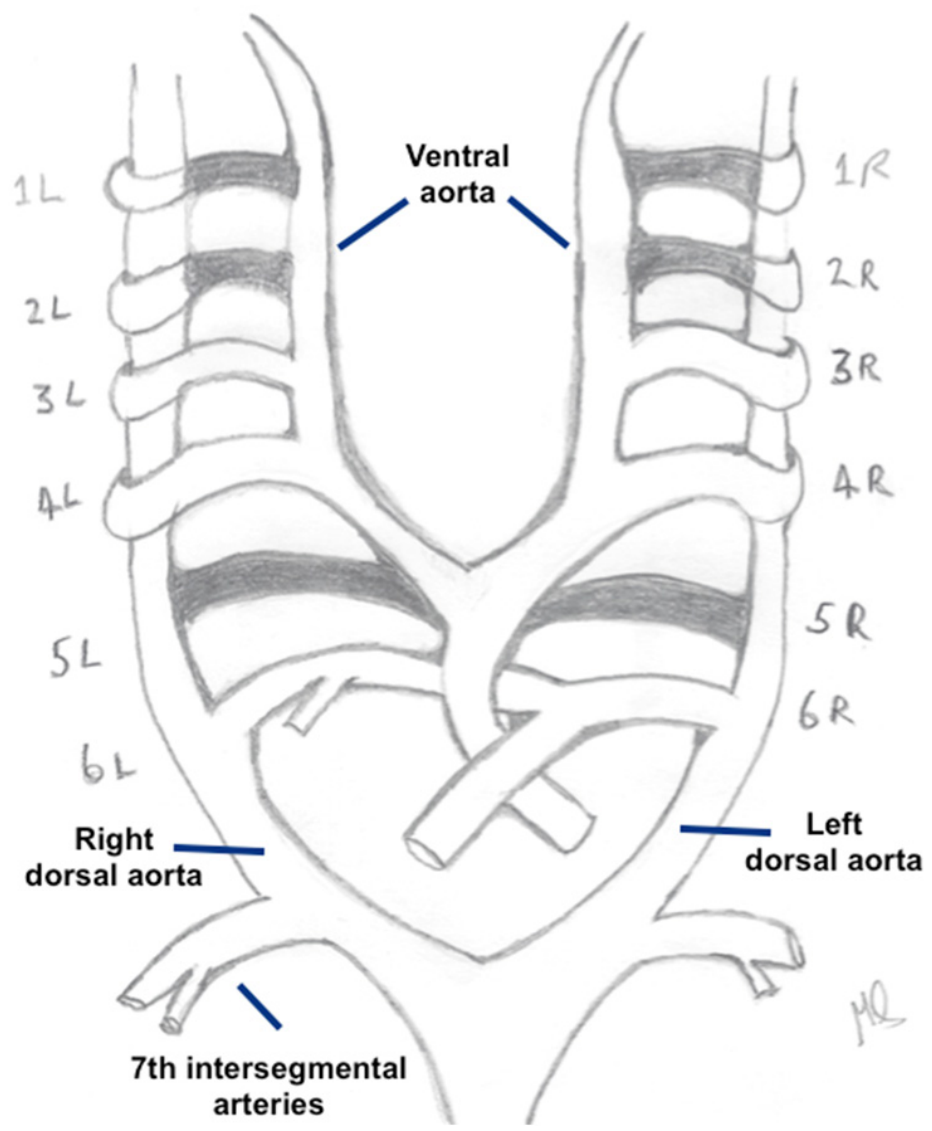

Figure 5. Classic Rathke's diagram representing embryological development of aortic arches

aortic arch; in circumflex aorta, the dorsal aorta contralateral to the aortic arch is the one that persists in life $^{[40]}$. Therefore, based on this hypothetical model, involutions that occur in circumflex aorta with right aortic arch are (1) the right sixth aortic arch; and (2) the left fourth aortic arch with persistence of the left sixth aortic arch and left dorsal aorta (dorsal aortic root segment 8). Alternatively, it may also share a common pathway with embryological formation of a cervical aortic arch or a double aortic $\operatorname{arch}^{[5,12,41]}$. In the latter, if obliteration between the left common carotid and subclavian arteries occurs early in-utero, the segment may completely involute giving rise to a true form of circumflex arch at birth ${ }^{[12]}$.

As with other types of aortic obstruction, hemodynamic mechanisms resulting in reduced flow in the ascending aorta during fetal life may contribute to the development of arch hypoplasia ${ }^{[5]}$. In addition, compression of the retrooesophageal component between oesophagus and spine during fetal development may also contribute. The aetiology of hypoplasia, when it occurs, is probably not genetic but rather acquired as a consequence of changes in fetal flow patterns.

\section{TERMINOLOGY}

The adjective 'circumflex' describes a structure that is curved or bends around another structure, in this case, the aortic arch bending posteriorly around the oesophagus. The term circumflex aorta and circumflex aortic arch are also used interchangeably and both terms have been used in the surgical literature to refer to the much more common form of circumflex aorta with right aortic arch.

The term circumflex aorta or circumflex aortic arch should be used as a generic term to refer to a congenital arch anomaly with high retroesophageal arch segment above the tracheal carina with both the 
proximal descending aorta and ductal ligamentum on the opposite side to the arch, to form a vascular ring. It only refers to the proximal segment of the descending aorta that is on the contralateral side of the aortic arch, and the subsequent descending aorta may or may not cross back to the other side of the spine. Not all retroesophageal arch is morphologically a circumflex arch, such as double aortic arch. A circumflex aorta can be described as a constellation of abnormal lie and development of both the aortic arch as well as the descending aorta, keeping in mind that the course of the descending aorta may have implications on therapeutic management strategies.

In describing circumflex aorta, the following need to be specified further: (1) the laterality of the arch either "circumflex aorta with right aortic arch" or "circumflex aorta with left aortic arch" - (2) the presence of any aberrant subclavian artery; (3) the caliber of the arch - unobstructed, or degree of hypoplasia, e.g., "Circumflex aorta with right aortic arch with aberrant left subclavian artery - unobstructed"; "Circumflex aorta with left aortic arch with aberrant right subclavian artery - critical hypoplasia". Such a morphological description will summarize the potential deleterious physiological effects of this anomaly on the patient: (1) a vascular ring that causes tracheal or esophageal compression; and (2) any degree of arch obstruction if present, which causes haemodynamic compromise.

\section{PATHOPHYSIOLOGY AND CLINICAL PRESENTATION}

The clinical manifestation of circumflex aortic arch is associated with pathophysiology that results from vascular compression; Kommerell's diverticulum; associated arch hypoplasia and intra-cardiac lesions. The constellation of this pathophysiology, as well as ductal patency, will therefore dictate the severity and timing of clinical presentation.

The hallmark of circumflex aortic arch is a high retrooesophageal arch segment. This crowds the mediastinal space posteriorly, and may result in aortic compression of the oesophagus and the trachea. Older children or adults can report long-standing, intermittent noisy breathing. Although severe airway symptoms are uncommon in young children, a sudden presentation with acute respiratory distress has been reported in infancy without major preceding symptoms ${ }^{[18]}$. The mechanism of acute deterioration is not clear but we postulate that this could due to closure of arterial duct and subsequent tightening of the vascular ring with ductal ligamentum. When compression is severe, the underlying tracheobronchomalacia could result in severe residual airway obstruction in the infant despite relief of vascular ring, which may persist despite full anatomical correction of circumflex aortic $\operatorname{arch}^{[7]}$.

Paediatric patients, who need intervention, usually present with significant airway symptoms ${ }^{[35]}$. Dysphagia is an uncommon presentation in children but likely to be under reported ${ }^{[19]}$. In the authors' experience, parents may describe intermittent "choking" when the infant starts to take solid food and some toddlers with a vascular ring develop an aversion to solid food.

Severe arch hypoplasia is uncommon, but could result in cardiovascular collapse during neonatal period or early infancy ${ }^{[5,29]}$. As opposed to the usual situation with coarctation of the aorta, the timing and mechanism of obstructive symptoms is much more akin to that of interruption of the aortic arch, as for type B aortic interruption, and the two may be very easily confused on echocardiographic assessment ${ }^{[5]}$. The hypoplastic distal circumflex aortic arch does not exhibit the dynamic obstruction that occurs with classical coarctation; however, treatment with prostaglandin to open and maintain ductal patency to the descending aorta is just as important in cases of severe arch hypoplasia presenting with signs of heart failure or shock and variably diminished pulses. Stenosis and hypoplasia in different sites or even multiple sites in patients with circumflex arch has been reported. When the base of aberrant subclavian is stenosed or obstructed, there will be differentiation of blood pressure between two arms; when presented without chest pain and diminished pulse in one arm, it simulates an asymptomatic chronic dissecting aortic aneurysm ${ }^{[42]}$. 
A small proportion of patients with circumflex aortic arch may remain asymptomatic or do not present until much later in life ${ }^{[11,16,43]}$. Asymptomatic circumflex arch may also present as an incidental finding with intracardiac lesion such as $\mathrm{VSD}^{[33]}$. However, the presence of a retroesophageal arch segment may result in a more severe form of vascular ring and symptoms may be more common in those with an unobstructive arch than in those with hypoplastic arch.

\section{IMAGING}

When presented incidentally, mediastinal widening on plain chest $\mathrm{x}$-ray in circumflex aortic arch can be mistaken as a mediastinal mass or an aortic aneurysm ${ }^{[8]}$. The proximal and distal ends of the retroesophageal transverse arch may form bilateral "aortic knobs" on chest x-ray resulting in a symmetrical superior mediastinal widening.

Barium swallow is useful to provide a clear demonstration of posterior oesophageal compression from the circumflex $\operatorname{arch}^{[17,22]}$. In severe oesophageal compression, there could reflux of the contrast materials into the nasopharynx ${ }^{[17]}$. Prior to the advent of CT, suspicious findings on barium study would trigger cardiac catheterization, leading to the diagnosis of circumflex aorta ${ }^{[17]}$.

Transthoracic echocardiography is the most common initial diagnostic test, which leads to the suspicion of congenital arch malformation. Echocardiography also further evaluates for any arch obstruction and intracardiac lesions. As a hypoplastic retroesophageal segment is not easily demonstrated on echocardiography, a circumflex aorta with right aortic arch, aberrant left subclavian artery, and critical arch hypoplasia can be easily mistaken for type B aortic arch interruption ${ }^{[5]}$.

$\mathrm{CT}$ angiogram with $3 \mathrm{D}$ reconstruction is the best imaging modality to demonstrate the presence of retroesophageal transverse arch and confirms the definitive diagnosis of circumflex aortic arch. A CT scan will also identify compression of the trachea and oesophagus [Figures 6 and 7].

In patients presenting with respiratory symptoms, bronchoscopy is often performed first by the otolaryngology team. The presence of pulsatile compression will then trigger cross-sectional imaging and establish the diagnosis of a circumflex arch. The diagnosis can be missed in the absence of pulsatile compression, which may not be easily evident in cases where there is complete occlusion of the main stem bronchus ${ }^{[9]}$. CT is, therefore, recommended to exclude vascular compression. When the diagnosis of circumflex aortic arch is established, intra-operative bronchoscopy may help to confirm adequate relief of vascular compression at the time of surgical repair.

\section{SURGICAL MANAGEMENT}

Surgery is indicated for significant symptoms and for any associated significant aortic arch hypoplasia. The surgical approach and timing of surgery are likely dictated by the severity of symptoms and arch obstruction. Complications associated with Kommerrell diverticulum, such as progressive aneurysm or dissection may also trigger for surgical intervention. Surgical management varies in literature, from simple division of the arterial ligament alone to full anatomical correction such as an aortic uncrossing procedure.

\section{Division of arterial ligament}

Without arch hypoplasia or obstruction, division of arterial ligament alone has been used to relieve the symptoms from vascular ring. Symptomatic improvement had been reported following division, although long-term data are lacking ${ }^{[18]}$. Nevertheless, this approach may offer a simple surgical solution associated with low surgical morbidity, without needing cardiopulmonary bypass or extensive posterior mediastinal dissection. Surgery can be approached via a standard posterolateral thoracotomy, or less invasive procedure 


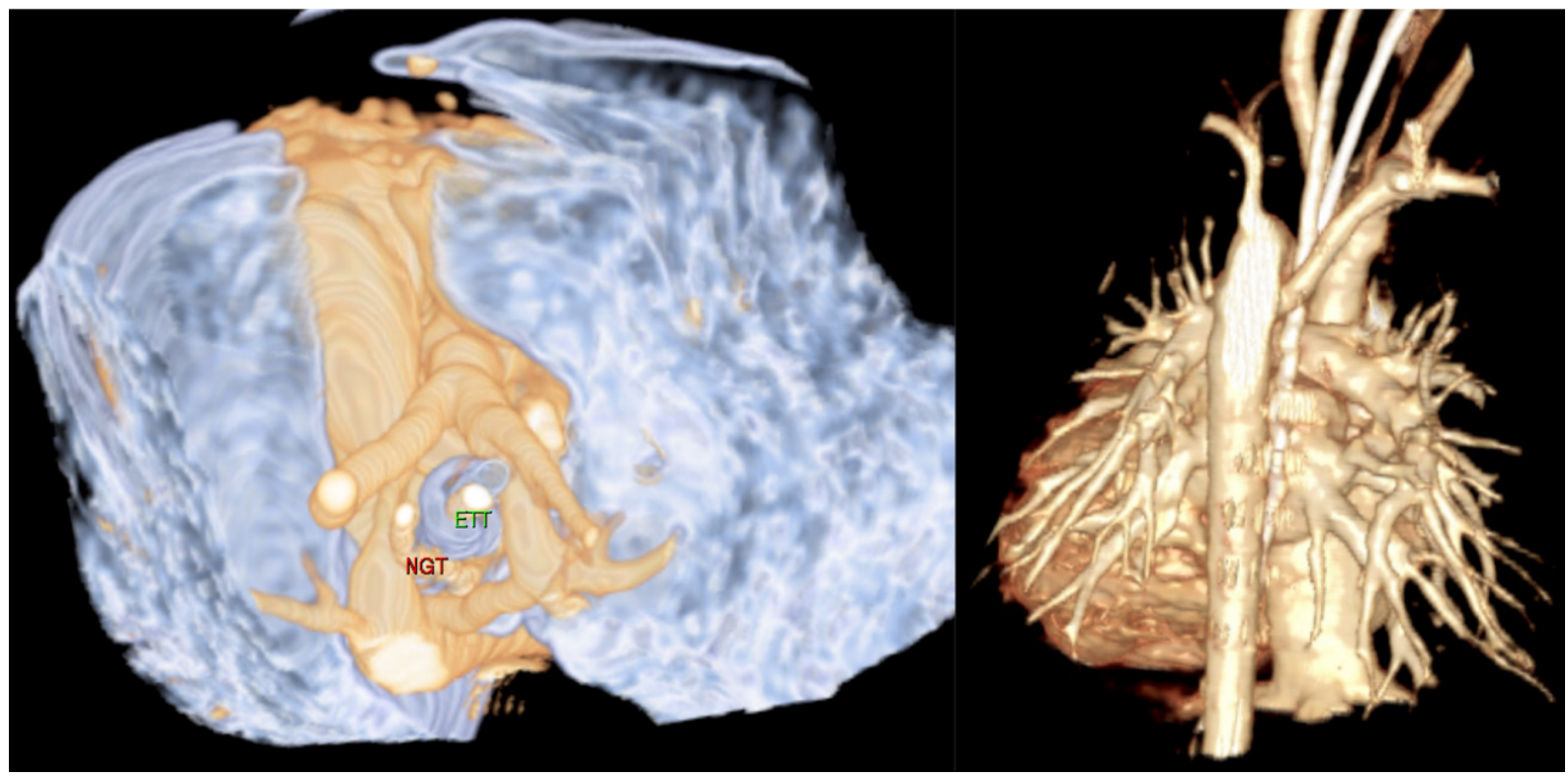

Figure 6. Computed tomography scan demonstrating (left) cross-section aerial view of a circumflex aorta with right aortic arch, encircling the airway [endotracheal tube (ETT)] and oesophagus [nasogastric tube (NGT)]; and (right) posterior view of the same patient demonstrating the hypoplastic retroesophageal aortic arch segment

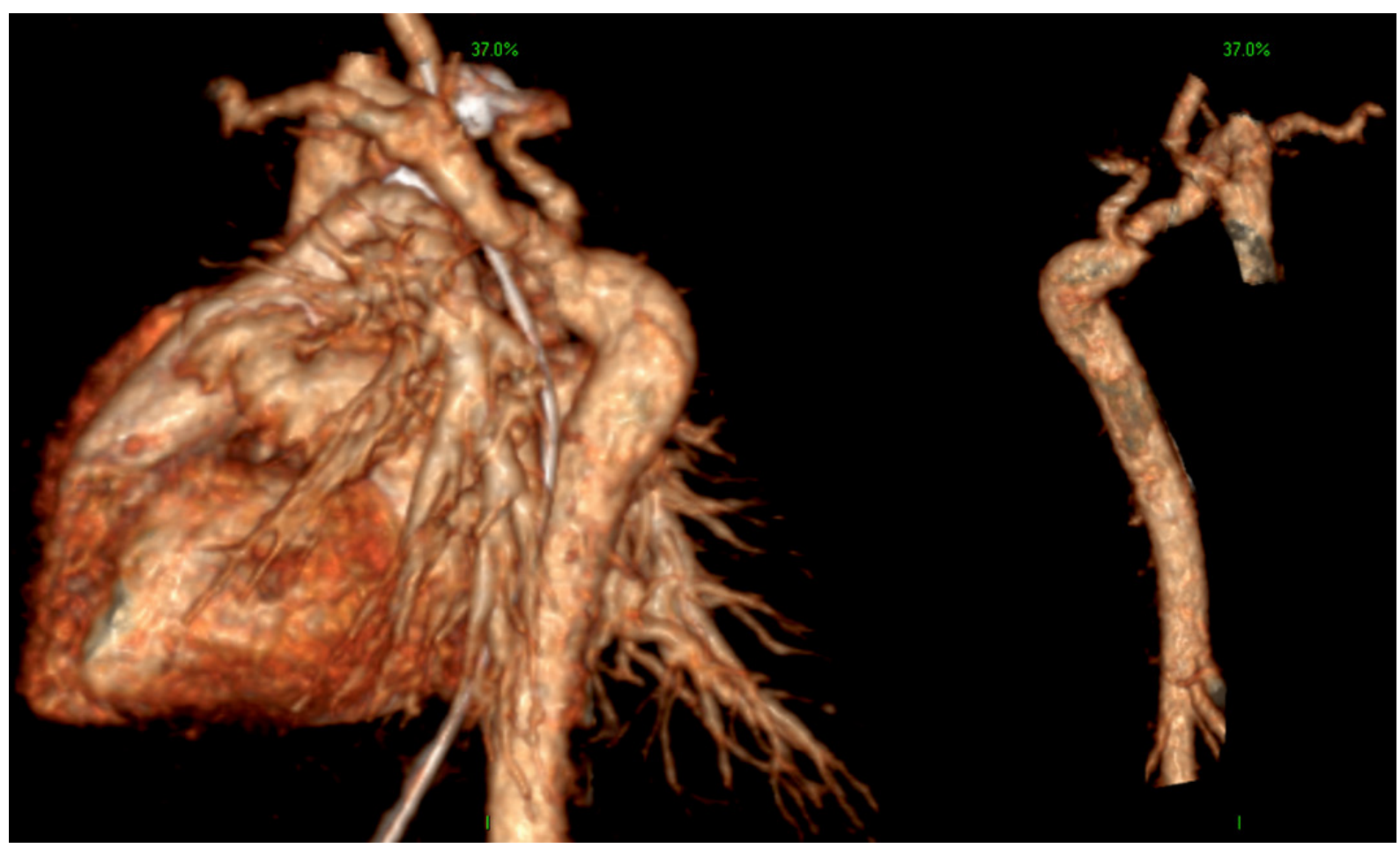

Figure 7. Computed tomography scan demonstrating (left) the left lateral view demonstrating a circumflex aorta with left aortic arch; and (right) anterior view of the same patient showing a circumflex aorta with left aortic arch and hypoplastic arch segment

such as posterior mini-thoracotomy, or video assisted thoracoscopic procedure. The arterial ligament is a structure that cannot be visualized in pre-operative imaging but its presence is presumed at the base of aberrant subclavian artery. 
Division of the arterial ligament alone, however, does not address compression of the mediastinal organs from the retroesophageal arch segment. In the absence of aortic arch hypoplasia, this approach may be justifiable in patients with mild symptoms or asymptomatic patients who underwent other cardiac or thoracic procedures ${ }^{[33]}$.

\section{Aortic arch uncrossing procedure}

Optimal symptomatic relief may not be achieved by dividing the arterial ligament alone due to persistent retroesophageal aortic arch compression. Therefore, the aortic uncrossing procedure was described by the French group in 1984 in three infants with circumflex aortic arch - all of whom had previously undergone ligamentum division alone but remained ventilator dependent ${ }^{[1,2]}$. These 3 patients were extubated after undergoing aortic uncrossing procedure; and became the subjects on three French reports of circumflex $\operatorname{arch}^{[1,2,14]}$.

The aortic uncrossing procedure is performed through a median sternotomy with cardiopulmonary bypass, hypothermia, and a short period of circulatory arrest. Deep hypothermic total circulatory arrest is used in the original and subsequent series ${ }^{[1,2,4]}$. The aorta is transected distal to the origin of the right subclavian artery and the proximal stump is over-sewn. The ligamentum arteriosum is ligated and divided. The retroesophageal aortic arch is dissected from its posterior attachments and brought anteriorly to the left side of the ascending aorta. An arteriotomy is performed on the side of the ascending aorta below the left carotid artery. An end to side anastomosis is performed. The right subclavian artery can be divided to facilitate mobilization of the arch anteriorly, although this is not mandator $y^{[4,14]}$. Selective antegrade cerebral perfusion with continuous cardiac perfusion and moderate hypothermia - avoiding total body circulatory arrest - is an alternative cardiopulmonary bypass strategy [Figure 8$]^{[6]}$.

\section{Posterior aortic translocation}

The surgical approach to address circumflex aorta with left aortic arch is less well described ${ }^{[7,9]}$. The aortic arch is on the left side, and therefore does not needs to be uncrossed to the "normal" side. In our own experience and of past reports, the proximal descending aorta is on the right but later courses back to the left ${ }^{[7]}$. Therefore, instead of "uncrossing" the arch, posterior aortic translocation is required. The presence of the right-sided location of the proximal descending aorta is the main issue, which requires translocation into the left chest. The division of the ligamentum relieves the ring and the translocation procedure removes the posterior airway compression. This procedure requires extensive mobilization of the entire descending thoracic aorta. In the presence of unobstructed arch, posterior aortopexy is performed. The procedure can be approached via left thoracotomy without cardiopulmonary bypass. In the presence of the arch hypoplasia, the retroesophageal segment can be resected and an extended end-to-end anastomosis can be performed [Figure 9] ${ }^{[7]}$.

\section{Aortic arch hypoplasia or obstruction}

Several surgical approaches have been reported for the management of circumflex aorta with hypoplastic arch. These include addressing the arch obstruction alone without correcting the circumflex anatomy resection of the coarctation, relieving the obstruction either using a patch or placing an extra anatomic graft. However, this approach will leave a complex vascular ring in situ which could be a problematic issue in the future with persistent oesophageal and airway compression.

Full anatomical correction of circumflex aorta will therefore require the following procedures: circumflex aorta with hypoplastic right aortic arch: resection of the hypoplastic segment, aortic arch uncrossing procedure and end-to-side anastomosis to the aorta anteriorly. Cardiopulmonary bypass with deep hypothermic total circulatory arrest can be used; but we preferred the strategy of selective antegrade cerebral perfusion via the innominate artery perfused via a $3 \mathrm{~mm}$ to $3.5 \mathrm{~mm}$ Goretex shunt ${ }^{[6]}$. Aortic 


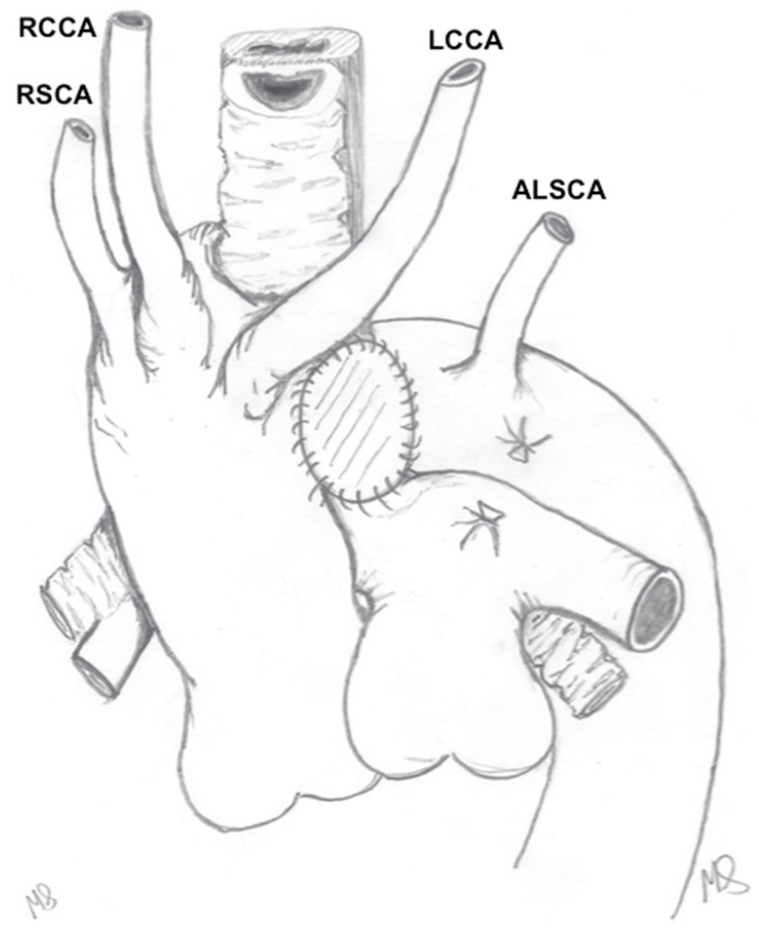

Figure 8. Sketch demonstrating the aortic uncrossing procedure with reimplantation (with patch augmentation) of the retroesophagal arch anteriorly on the left side of the ascending aorta. The head and neck vessels are: left common carotid artery (LCCA), right common carotid artery (RCCA), right subclavian artery (RSCA) and the aberrant left subclavian artery (ALSCA)

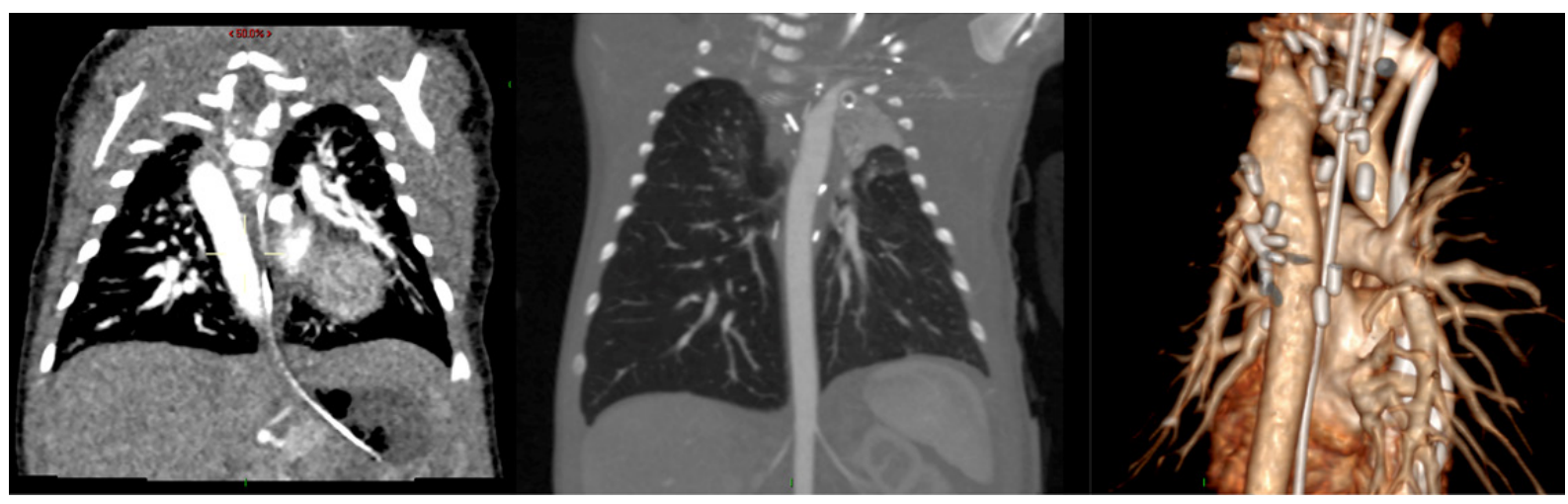

Figure 9. Computed tomography scan of a neonate with circumflex aorta with left aortic arch and critical arch hypoplasia: (left) descending aorta location to the right side of the spine; (middle) normalization of the course of the descending aorta following posterior aortic translocation; (right) 3D reconstruction of the aortic arch following surgery

uncrossing procedure has also been used in single ventricular physiology with aortic arch obstruction requiring a concomitant Norwood procedure ${ }^{[34]}$.

Circumflex aorta with hypoplastic left arch: a rare variant of obstructed circumflex aorta. Resection of a long hypoplastic segment, posterior aortic translocation, division of the arterial ligament, and extended end to end repair of the arch has been described ${ }^{[7]}$. These surgical strategies addressed all the associated pathophysiological mechanisms of circumflex aorta with hypoplastic arch: (1) relief of vascular ring; (2) removal of posterior arch compression on the trachea; and (3) correction of any arch obstruction. If the proximal arch (between innominate and left common carotid artery) is adequate, the procedure can be performed off-pump from the left chest. 


\section{Post-operative issues}

Due to its proximity, recurrent laryngeal nerve injury is a reported post-operative complication especially when a more extensive procedure such as aortic uncrossing surgery is required ${ }^{[4,14]}$. We had not encountered this complication in our reported surgical experience in both circumflex right and left aortic $\operatorname{arch}^{[6,7]}$. Other complications associated with arch surgery include phrenic nerve injury and chylothorax.

Persistent respiratory symptoms can be due to (1) residual compression from retroesophageal arch if only arterial ligament division alone was performed; and/or (2) underlying tracheobronchomalacia. In children who presented early in the neonatal period or infancy, underlying tracheomalacia could be severe and persistent even after total anatomical correction ${ }^{[7]}$. Failure to extubate will require tracheostomy and a home ventilator may be required. Posterior tracheobronchopexy has been used following repair of hypoplastic circumflex aortic arch to facilitate extubation and avoid tracheostomy and long-term ventilation ${ }^{[7]}$. Concomitant circumflex aortic arch repair with posterior tracheopexy has been reported recently by the Boston group $^{[35]}$.

\section{CONCLUSION}

The role of surgical intervention in the management of circumflex aorta is evolving. The prevalence of functionally circumflex aorta and persistent symptoms following double aortic arch repair remains to be defined. The aortic arch uncrossing procedure provides full anatomical correction for circumflex aorta with right aortic arch, but requires hypothermic circulatory arrest, and therefore considerable debate still exists if this is always required in the absence of arch obstruction. Undoubtedly, the aortic arch uncrossing procedure has an important role as a rescue strategy in patients with significant residual symptoms postoperatively but its role as a primary strategy is also emerging for severely symptomatic patients, especially with improvised technique to minimize operative morbidities. Posterior aortic translocation also provides full anatomical correction in circumflex variants with appropriate anatomy, without the need of arch uncrossing. When arch obstruction is present, full anatomical repair with aortic uncrossing or posterior aortic translocation should always be advocated to relieve arch obstruction as well as to correct the pathological effects of circumflex aorta.

\section{DECLARATIONS}

\section{Acknowledgments}

The authors are extremely grateful to Miss Mhari Chambers, RGN for all her drawings in this manuscript.

\section{Authors' contributions}

Made substantial contributions to conception and design of the study, performed literature review and preparation of the manuscript: Bader V, Peng E

Made substantial contributions to conception and design of the study and manuscript review: Knight WB, Danton $\mathrm{MH}$

\section{Availability of data and materials}

Not applicable.

Financial support and sponsorship

None.

\section{Conflicts of interest}

All authors declared that there are no conflicts of interest.

\section{Ethical approval and consent to participate}

Not applicable. 


\section{Consent for publication}

Patient consents were sought for publication of cross-section images used as figures in this manuscript.

\section{Copyright}

(c) The Author(s) 2020.

\section{REFERENCES}

1. Bruniaux J, Villain E, Sidi D, et al. Severe tracheo-bronchial compression caused by a circumflex aorta. Apropos of 3 cases treated by aortic uncrossing. Arch Mal Coeur Vaiss 1984;77:557-63.

2. Planché C, Lacour-Gayet F. Aortic uncrossing for compressive circumflex aorta. 3 cases. Presse Medicale Paris Fr 1983 1984;13:1331-2.

3. Backer CL, Mongé MC, Russell HM, Popescu AR, Rastatter JC, Costello JM. Reoperation after vascular ring repair. Semin Thorac Cardiovasc Surg Pediatr Card Surg Annu 2014;17:48-55.

4. Russell HM, Rastatter JC, Backer CL. The aortic uncrossing procedure for circumflex aorta. Oper Tech Thorac Cardiovasc Surg 2013;18:15-31.

5. Knight WB. Hypoplastic right retro-oesophageal aortic arch: similarities to interrupted aortic arch. Br Heart J 1989;62:477-81.

6. Bader V, Walayat M, Smith B, Davidson M, Morue S, Danton MHD. Circumflex retroesophageal aorta mimicking aortic interruption: a rare cause of aortic obstruction in a neonate. World J Pediatr Congenit Heart Surg 2014;5:599-602.

7. Peng E, McAllister K, Walker A, Savage T, Hunter L. Surgical approach for hypoplastic mirror circumflex aortic arch. Ann Thorac Surg 2019;107:e313-5.

8. Shuford W, Sybers R, Gordon I, Baron M, Carson G. Circumflex retroesophageal right aortic arch simulating mediastinal tumor or dissecting aneurysm. Am J Roentgenol 1986;146:491-6.

9. Baird CW, Prabhu S, Buchmiller TL, Smithers C, Jennings R. Direct tracheobronchopexy and posterior descending aortopexy for severe left mainstem bronchomalacia associated with congenital pulmonary airway malformation and left circumflex aortic arch. Ann Thorac Surg 2016;102:e1-4.

10. Annan JL. Case of an abnormal sinuous aorta. J Anat Physiol 1910;44:241-3.

11. De Balsac RH. Left aortic arch (posterior or circumflex type) with right descending aorta. Am J Cardiol 1960;5:546-50.

12. Edwards JE. Anomalies of the derivatives of the aortic arch system. Med Clin North Am 1948;32:925-49.

13. Hastreiter AR, D'Cruz IA, Cantez T, Namin EP, Licata R. Right-sided aorta. I. Occurrence of right aortic arch in various types of congenital heart disease. II. Right aortic arch, right descending aorta, and associated anomalies. Br Heart J 1966;28:722-39.

14. Robotin MC, Bruniaux J, Serraf A, et al. Unusual forms of tracheobronchial compression in infants with congenital heart disease. $J$ Thorac Cardiovasc Surg 1996;112:415-23.

15. Paul RN. A new anomaly of the aorta; left aortic arch with right descending aorta. J Pediatr 1948;32:19-29.

16. Mowitz S. Left am-tic arch and right descending aorta. Am J Cardiol 1962;6.

17. Berman W, Yabek SM, Dillon T, Neal JF, Akl B, Burstein J. Vascular ring due to left aortic arch and right descending aorta. Circulation 1981;63:458-60.

18. Ergin MA, Jayaram N, LaCorte M. Left aortic arch and right descending aorta: diagnostic and therapeutic implications of a rare type of vascular ring. Ann Thorac Surg 1981;31:82-5.

19. Murthy K, Mattioli L, Diehl AM, Holder TM. Vascular ring due to left aortic arch, right descending aorta, and right patent ductus arteriosus. J Pediatr Surg 1970;5:550-4.

20. Planché C, Roussin R, Paul JF, Belli E, Serraf A. A rare form of aortic arch anomaly: left aortic and right descending aortic arch. Arch Mal Coeur Vaiss 2004;97:549-53.

21. Watanabe M, Minami K. Left aortic arch with right descending aorta and right ligamenturn arteriosum associated with d-TGA and large VSD: surgical treatment of a rare form of vascular ring. J Pediatr Surg 1995;30:1363-5.

22. Whitman G, Stephenson LW, Weinberg P. Vascular ring: left cervical aortic arch, right descending aorta, and right ligamentum arteriosum. J Thorac Cardiovasc Surg 1982;83:311-5.

23. Backer CL, Mongé MC, Popescu AR, Eltayeb OM, Rastatter JC, Rigsby CK. Vascular rings. Semin Pediatr Surg 2016;25:165-75.

24. Tanaka A, Milner R, Ota T. Kommerell's diverticulum in the current era: a comprehensive review. Gen Thorac Cardiovasc Surg 2015;63:245-59.

25. Cinà CS, Althani H, Pasenau J, Abouzahr L. Kommerell's diverticulum and right-sided aortic arch: a cohort study and review of the literature. J Vasc Surg 2004;39:131-9.

26. Kommerell B. Verlagerung des osophagus durch eine abnorm verlaufende arteria subclavia dextra (Arteria lusoria). Fortschr Roentgenster 1936;54:590-5.

27. Luciano D, Mitchell J, Fraisse A, Lepidi H, Kreitmann B, Ovaert C. Kommerell diverticulum should be removed in children with vascular ring and aberrant left subclavian artery. Ann Thorac Surg 2015;100:2293-7.

28. Bein S, Saba Z, Patel H, Reinhartz O, Hanley FL. Coarctation of the aorta in the right aortic arch with left aberrant innominate artery. Pediatr Cardiol 2006;27:621-3.

29. Song J, Kim WH, Kim H, et al. Repair of coarctation in right circumflex retroesophageal arch. Ann Thorac Surg 2009;87:307-9.

30. Nisli K, Aydogan U, Tansel T. Left-sided aortic arch, right-sided descending aorta, and right-sided arterial duct associated with 
interruption of the aortic arch and presence of Abbott's artery. Cardiol Young 2007;17:445-7.

31. Abbott ME. Coarctation of the aorta of the adult type II. A statistical study and historical retrospect of 200 recorded cases, with autopsy, of stenosis or obliteration of the descending arch in subjects above the age of two years. Am Heart J 1928;3:381-421.

32. Lerberg DB. Abbott's artery. Ann Thorac Surg 1982;33:415-6.

33. Tissot C, Didier D, Beghetti M, Kalangos A, Myers PO. Asymptomatic right circumflex aortic arch associated with ventricular septal defect and biscuspid aortic valve. Heart Surg Forum 2015;18:114.

34. Bryant R, Wallen W, Rizwan R, Morales DL. Modified aortic uncrossing procedure: a novel approach for norwood palliation of complex univentricular congenital heart disease with a circumflex aorta. World J Pediatr Congenit Heart Surg 2017;8:507-10.

35. Kamran A, Friedman KG, Jennings RW, Baird CW. Aortic uncrossing and tracheobronchopexy corrects tracheal compression and tracheobronchomalacia associated with circumflex aortic arch. J Thorac Cardiovasc Surg 2020; doi: 10.1016/j.jtcvs.2020.03.158.

36. Backer CL, Bharadwaj SN, Eltayeb OM, Forbess JM, Popescu AR, Mongé MC. Double aortic arch with kommerell diverticulum. Ann Thorac Surg 2019;108:161-6.

37. Kleine P, Balci M, Moritz A. Primary complete repair of partial double aortic arch and kommerell diverticulum. Ann Thorac Surg 2011;91:627-9.

38. Sierra-Galan LM, Shveid-Gerson D, Gomez-Garza G, Richheimer-Wohlmuth RM, Rey-Rodriguez A. Double incomplete aortic arch and Kommerell's diverticulum as a cause of chronic cough. Arch Cardiol México 2015;85:158-60.

39. Stojanovska J, Cascade PN, Chong S, Quint LE, Sundaram B. Embryology and imaging review of aortic arch anomalies. $J$ Thorac Imaging 2012;27:73-84.

40. Priya S, Thomas R, Nagpal P, Sharma A, Steigner M. Congenital anomalies of the aortic arch. Cardiovasc Diagn Ther 2018;8:S26-44.

41. Hanneman K, Newman B, Chan F. Congenital variants and anomalies of the aortic arch. Radio Graphics 2017;37:32-51.

42. Shuford W, Sybers R, Gordon I, Baron M, Carson G. Circumflex retroesophageal right aortic arch simulating mediastinal tumor or dissecting aneurysm. Am J Roentgenol 1986;146:491-6.

43. Edwards JE. Retro-esophageal segment of the left aortic arch, right ligamentum arteriosum and right descending aorta causing a congenital vascular ring about the trachea and esophaguw. Proc Staff Meet Mayo Clin 1948;23:108-16. 